\title{
Noninvasive Sensing Techniques and Geophysical Methods for Cultural Heritage and Civil Infrastructures Monitoring
}

\author{
Francesco Soldovieri, ${ }^{1}$ Jean Dumoulin, ${ }^{2}$ Nicola Masini, ${ }^{3}$ and Erica Utsi ${ }^{4}$ \\ ${ }^{1}$ Istituto per il Rilevamento Elettromagnetico dell'Ambiente, Consiglio Nazionale delle Ricerche, Via Diocleziano 328, \\ 80124 Napoli, Italy \\ ${ }^{2}$ Monitoring, Assessment and Computational Science Department (MACS), French Institute of Science and Technology for Transport, \\ Development and Networks (IFSTTAR), Route de Bouaye, CS4, 44344 Bouguenais, France \\ ${ }^{3}$ Istituto per i Beni Archeologici e Monumentali, CNR, C.da S. Loja, 85050 Tito Scalo (PZ), Italy \\ ${ }^{4}$ Utsi Electronics Ltd., Cambridge CB22 7NZ, UK
}

Correspondence should be addressed to Francesco Soldovieri, soldovieri.f@irea.cnr.it

Received 24 January 2012; Accepted 24 January 2012

Copyright () 2011 Francesco Soldovieri et al. This is an open access article distributed under the Creative Commons Attribution License, which permits unrestricted use, distribution, and reproduction in any medium, provided the original work is properly cited.

This special issue presents a good and interesting review of the state of art and novel methodologies, instrumentation, and data-processing approaches to perform non- or minimally destructive investigations with a specific focus to the applicative field of the cultural heritages and critical infrastructure monitoring. In addition, it is concerned with some interesting theoretical and "in real world" examples of how the integration between the classical geophysical techniques with new emerging surface and subsurfacesensing techniques enables a multidepth, multiresolution, and multiscale monitoring.

P. L. Cosentino et al. present the new concept of "microgeophysics" of relevant interest in both the fields of the civil engineering and cultural heritage studies. In particular, the paper is concerned with research efforts in the field of the instrumentation and methodologies so to gain the relevant applicative advantages of time reduction without any loss of information, by taking care to the adoption of multichannel systems.

L. Fragasso and N. Masini present a very interesting use of the infrared reflectography in cultural heritage; in particular, the survey regards the assessment of the polyptych panel attributed to Vivarini's workshop conserved in the "Sigismondo Castromediano" Museum in Lecce. An effort has been made in processing the IR images through Principal Component Analysis and spectral indices able to give information about different pictorial drafting and restoration works.
L. N. Eppelbaum gives an interesting review of the microgravity investigations with a focus to archaeological applications, which is a very challenging research field. In particular, an advanced methodology based on gravity anomalies analysis and 3D modelling is presented with the aim to delineate ancient targets. The performed model computations indicate that microgravity investigations might be successfully applied at least in 20-25\% of archaeological sites in Israel.

Orlando et al's paper is concerned with the application of a multisensing approach based on geology, GPS, surveys of cracking, boreholes, seismic refraction and electrical resistivity tomography - to image the shallow stratigraphy and hypothesize the cause of instability of an historical centre. The synergy due to the combination of different geophysical surveys permits to depict underground features at small and medium scale.

The paper from G. Scardozzi et al. regards the contribution of the high-resolution multitemporal optical satellite imagery for change detection of a territory with a focus to the modifications that affected archaeological features and the landscape. In particular, the investigation was focused to five great Assyrian capital cities (Ashur, KarTukulti-Ninurta, Kalhu, Dur-Sharrukin, and Nineveh, in northern Iraq) by using very high-resolution satellite images taken between 2001 and 2007 and panchromatic photographs taken by U.S. spy satellites operating between 1965 and 1969. 
The paper by G. Bitelli et al. is an example of integrated surveying and monitoring activities for the control of an ancient structure, the Casalecchio di Reno sluice, located near Bologna, Italy, representing a case of "industrial archaeological site." Classical topography, high-precision spirit levelling, terrestrial laser scanning, digital close-range photogrammetry, and thermal imagery were used to monitor the stability and the movements of the structure. In addition, the creation of a 3D model of the structure was achieved and permitted to compare two situations, before and after the serious damages suffered by the sluice. Also, an analysis of the whole sluice, carried out at a regional scale, was done via the use of aerial photogrammetry.

The paper by R. Bernini et al. is concerned with the application of a new diagnostic technique for long-range strain and temperature monitoring, exploiting a a distributed fiberoptic sensor portable system based on Brillouin scattering. The experiment in laboratory has demonstrated the effectiveness of this novel technique as a structural assessment tool by measuring the strain on a $4 \mathrm{~m}$ long concrete beam and detecting the formation of a crack in the beam resulting from the external applied load.

Paper by A. Loperte et al. deals with an interesting case of use of the Ground Penetrating Radar (GPR) in monitoring of critical infrastructure by a high-resolution diagnostics. In particular, in this paper, the application of GPR has permitted the monitoring and diagnostics of one of the largest dams in the Basilicata region (Southern Italy) for safety management; in fact, the investigation had the aim to detect and localize underground sandstone banks that are potential ways of flow of water below the dam.

Paper by M. Marchetti et al. is concerned with the study of the performance of an infrared camera for the determination of ice road susceptibility, to build a new winter risk index, to improve the measurements rate, and to analyze its consistency with seasons and infrastructures environment. The comparison of this technique with the classical radiometer sensing technique shows a good effectiveness of the IR technique with promising perspectives.

Paper by S. Chambon et al. regards the use of automatic analysis of optical images for crack detection on the French national roads. The work gives three main contributions as: a state of the art of the image-processing tools applied to civil engineering, an approach based on multi-scale extraction and a Markovian segmentation for a fine-defect detection in pavement surface, the design and the validation of a protocol for evaluating the road pavement crack detection.

Paper by A. Palombo et al. regards an experiment at Montagnole site performed in the framework of FP7 ISTIMES project. Ground-based microwave radar interferometer and high-frequency InfraRed Thermography (IRT) were used at the same time to detect and characterize the dynamic displacement of a $16 \mathrm{~m}$ concrete beam affected by high-energy direct impacts of an iron ball weighting few tons. The results achieved by the two techniques were compared in terms of frequency analysis and show a very good correlation, which makes confident about the reliability of the diagnosis results.
The paper by G. Colangelo and A. Guariglia discusses a hazard assessment strategy and risk mitigation for rockfalls in a section of a national road, along the coast of Maratea (Southern Italy), using LIDAR technique and spatial modelling. The results represent a valid cognitive support to choose the most appropriate technical solution for topography strengthening and an example of good practice for the cooperation between innovative technologies and field emergency management.

The paper by S. Nordebo et al. represents a methodological contribution to the data fusion problem, which is of great interest in the integration of different techniques for critical infrastructure monitoring. In particular, it is presented a maximum likelihood-based approach to data fusion for electromagnetic (EM) and electrical resistive (ER) tomography. As a multiphysics problem formulation with applications in geophysics, the problem of tunnel detection based on EM and ER tomography is studied in this paper.

The paper by L. Pajewski et al. falls in the framework of the research activities for the development of physical-based data processing for GPR measurements. The effectiveness of the full-wave technique for the solution of the twodimensional plane-wave scattering problem is shown for a set of perfectly conducting and dielectric cylinders buried in a dielectric half-space, or in a finite-thickness slab. In particular, metallic pipes and air cavities are simulated as buried utilities.

The last paper by A. C. D. Royal et al. presents the progress of a research project that aims to develop a multisensor geophysical platform that can improve the probability of detection of the infrastructure buried beneath the carriageway. The multisensor platform is being developed in conjunction with a knowledge-based system that aims to provide information on how the properties of the ground might affect the sensing technologies being deployed. The fusion of data sources (sensor data and utilities record data) is also being researched to maximize the probability of location. This paper describes the outcome of the initial phase of testing along with the development of the knowledge-based system and the fusion of data to produce utility maps.

Francesco Soldovieri
Jean Dumoulin
Nicola Masini
Erica Utsi 

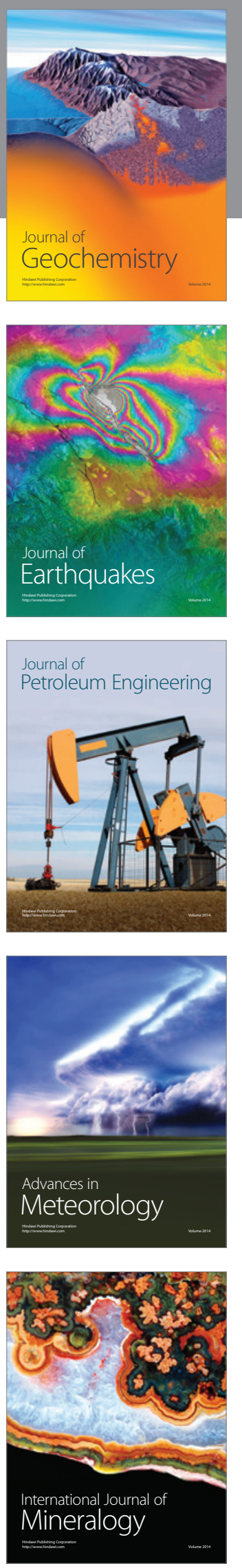
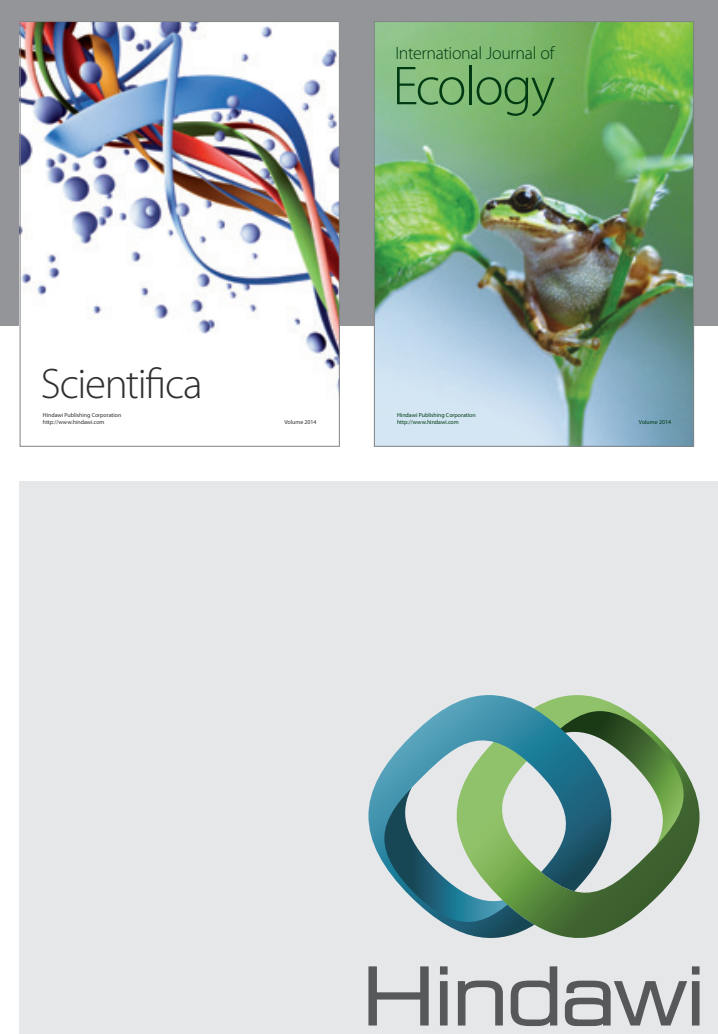

Submit your manuscripts at http://www.hindawi.com
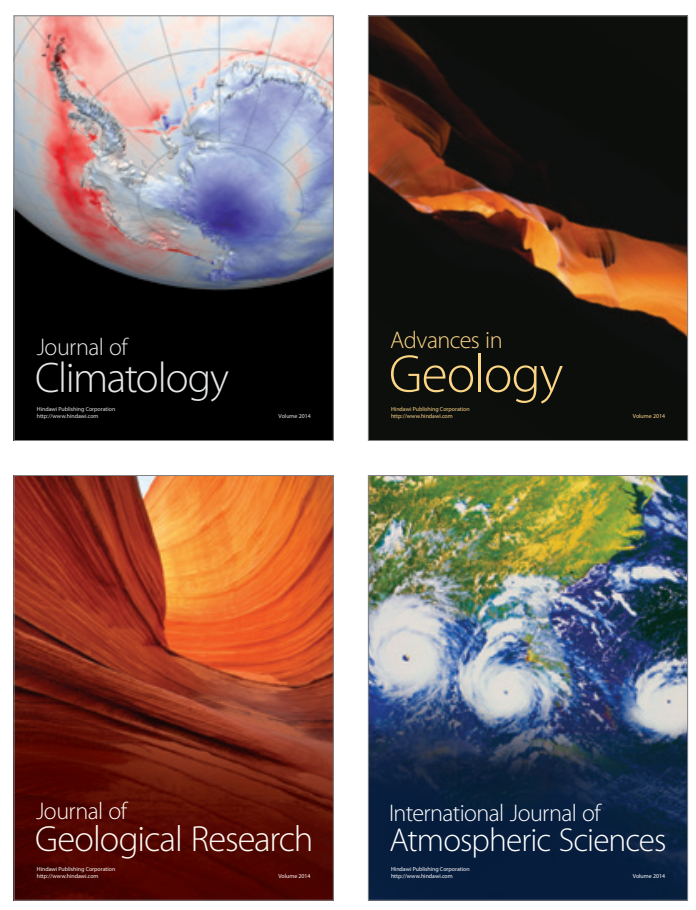
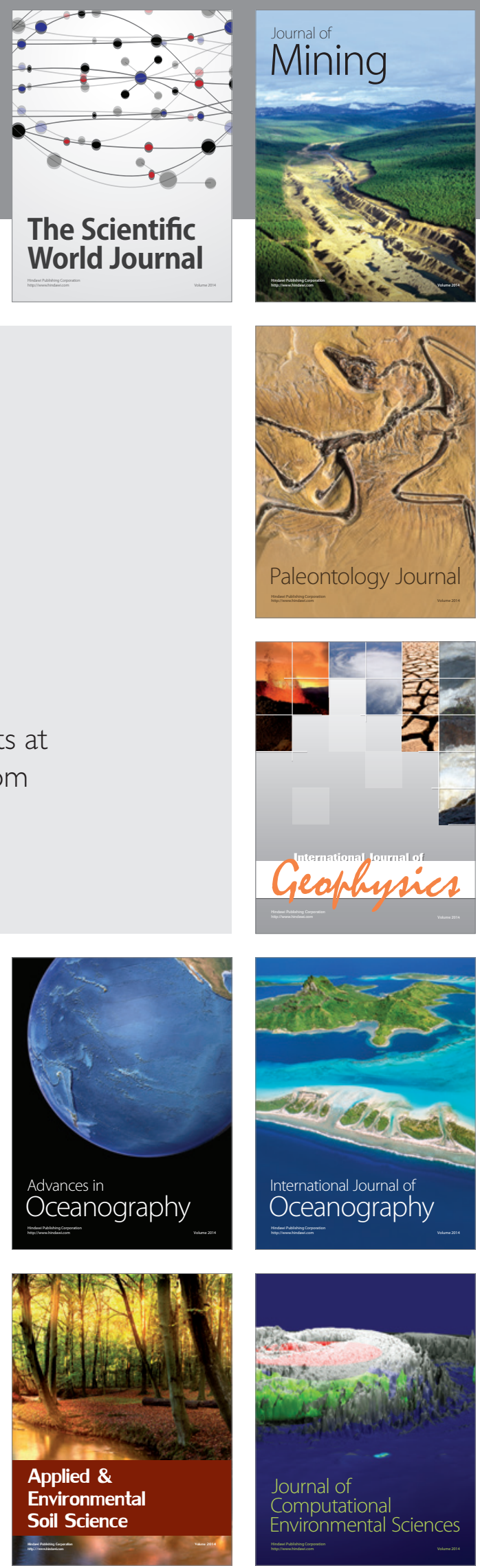